\title{
Electrophoretic Karyotypes and Chromosome Numbers in Candida Species
}

\author{
By BEATRICE B. MAGEE AND P. T. MAGEE* \\ Department of Microbiology, Michigan State University, East Lansing, \\ Michigan 48824-1101, USA
}

(Received 16 June 1986; revised 29 August 1986)

The electrophoretic karyotypes of five Candida albicans isolates and of five other Candida species have been determined, using orthogonal field alternating gel electrophoresis (OFAGE). None of the C. albicans isolates had the same electrophoretic karyotype. By comparing all five strains, we arrived at a chromosome number of nine to ten, but since the organism is diploid, we cannot distinguish genetically different chromosomes from homologues which resolve. We determined minimal chromosome numbers of 9 for Candida stellatoidea, 10 for C. glabrata and 6 for $C$. guilliermondii.

\section{INTRODUCTION}

Candida albicans is a dimorphic fungus which is the single most important fungal human pathogen (Odds, 1979). Its diploid nature (Whelan et al., 1980), combined with its lack of a sexual cycle, makes it a difficult subject for genetic analysis. Nevertheless, parasexual analysis, mediated by spheroplast fusion, has led to the accumulation of a reasonable amount of information about the genetics of the organism. A number of alleles of various auxotrophic markers have been isolated (Kakar et al., 1983; Poulter et al., 1981; Sarachek et al., 1981) and five linkage groups have been identified (Poulter \& Hanrahan, 1983; Poulter et al., 1981; Sarachek et al., 1981; Crandall, 1982; Kakar et al., 1983) using mitotic recombination and chromosome loss. The absence of meiotic segregation and the lack of cytogenetics has made assignment of the various linkage groups to chromosomes very difficult. Recently, the technique of orthogonal field alternating gel electrophoresis (OFAGE) has been developed to separate very large pieces of DNA (Carle \& Olson, 1984; Schwartz \& Cantor, 1982). This technique has allowed the visualization of 16 chromosomes in Saccharomyces cerevisiae, a number which corresponds to the genetically determined number of centromeres (Carle \& Olson, 1985). We have adapted this technique to separate the chromosomes of various Candida species, including several strains of $C$. albicans.

\section{METHODS}

Yeast strains. Strains used in these experiments included Sacch. cerevisiae strains BB4 (MATa leu2, his3, arg4), and W66-8a (homothallic, ura3, trp5, ade2, met4, leul).

C. albicans strains were clinical isolates: FC18 (Whelan et al., 1980), NUM63 (T. Suzuki, Nagoya University Medical School), SG483 (a derivative of ATCC 1026), 1840 (M. Gurwith, this Department) and SC5314 (Myra Kurtz, The Squibb Institute for Medical Research).

Representative strains of Candida glabrata (81/011), Candida tropicalis (81/019) and Candida parapsilosis (81/042) were provided by Dr Frank Odds (University of Leicester, Leicester, UK). Dr Alvin Rogers of this department supplied Candida stellatoidea CP5Am5. A strain of C. guilliermondii, NUM20, came from Nagoya University and was provided by T. Suzuki. Schizosaccharomyces pombe $\left(\mathrm{h}^{-}\right.$leul-32) came from Dr P. Nurse.

Abbreviation: OFAGE, orthogonal field alternating gel electrophoresis. 


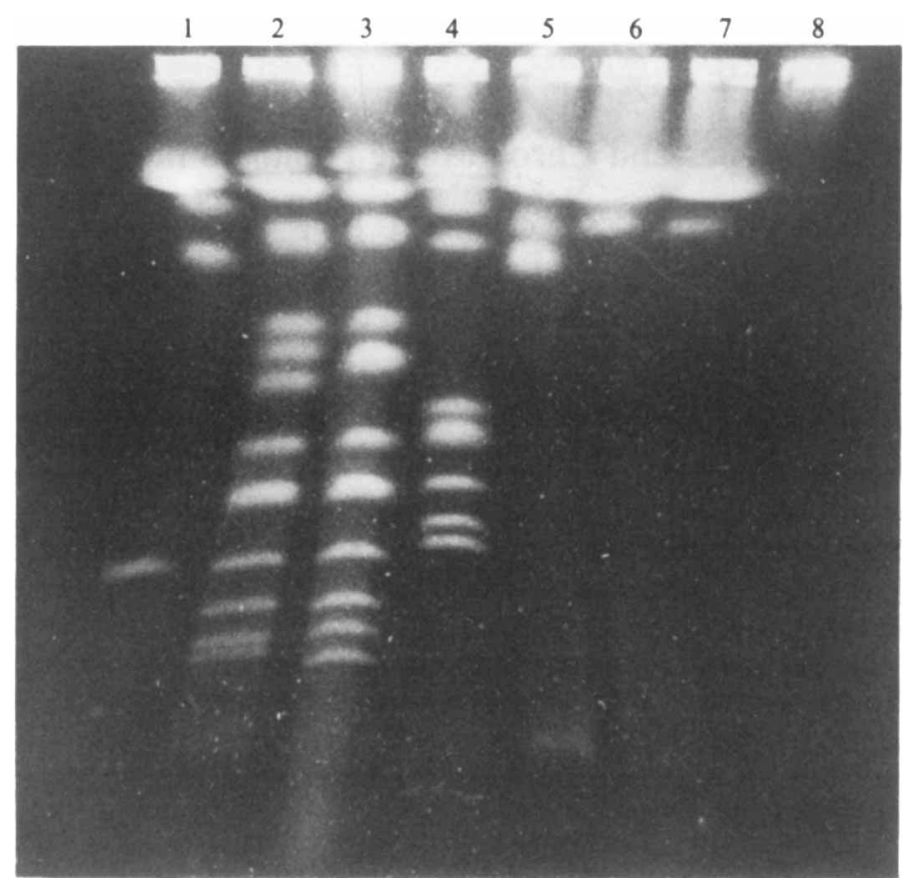

Fig. 1. DNA of various yeast strains was run on a $1.5 \%$ agarose gel, at a $60 \mathrm{~s}$ pulse rate at $300 \mathrm{~V}$ for $21 \mathrm{~h}$. Lane 1, Candida guilliermondii NUM20; lane 2, Sacch. cerevisiae BB4; lane 3, Sacch. cerevisiae W66-8a; lane 4,C. glabrata 81/011; lane 5, C. parapsilosis 81/042; lane 6, Candida albicans FC18; lane 7, $C$. tropicalis 81/019; lane 8, Schizosacch. pombe $\mathrm{h}^{-}$leul-32.

OFAGE. Chromosomes were prepared by a modification described by Carle \& Olson (1985) of the embeddedagarose procedure of Schwartz \& Cantor (1982). Agarose gels $(60 \mathrm{ml})$ were run on an OFAGE apparatus made by PlasLabs, Lansing, Mich., USA, according to the specifications of Carle \& Olson (1984). The $1.5 \%(\mathrm{w} / \mathrm{v})$ gels were run for $2 \mathrm{l} \mathrm{h}$ at $300 \mathrm{~V}, 60 \mathrm{~s}$ switching time and the $1.2 \%$ and $1.0 \%$ gels for $21 \mathrm{~h}$ at $300 \mathrm{~V}, 70 \mathrm{~s}$ switching time. Gels were stained with $0.5 \mu \mathrm{g}$ ethidium bromide $\mathrm{ml}^{-1}$ for $15 \mathrm{~min}$ and destained for $30 \mathrm{~min}$ or more.

\section{RESULTS}

Yeasts of the genus Candida show highly varied electrophoretic karyotypes

The OFAGE technique can be modified empirically by varying the pulse rate and the agarose gel concentration to allow the separation of bigger or smaller DNA molecules. We decided to get some estimate of the size of the chromosomes of several Candida species by comparing them to the well-studied Sacch. cerevisiae electrophoretic karyotype. Fig. 1 shows that with the exception of $C$. glabrata, all the Candida species examined had a majority of chromosomes bigger than band 10 of Sacch. cerevisiae. It was therefore necessary to modify the protocol described by Carle $\&$ Olson (1985). After several trials, we decided to use a $70 \mathrm{~s}$ pulse time and $1.2 \%$ agarose. This technique gave the results shown in Fig. 2 for several Candida species. C. guilliermondii is haploid, and it has five resolvable bands on the run shown in Fig. 2(a), lane 1. However, Fig. $2(b)$ shows another run in which six bands are resolvable from this organism. We conclude that six is a more plausible chromosome number than 5. C. glabrata (Fig. 2a, lane 2) shows 10 resolvable bands, with the fourth one up clearly a doublet. Available evidence indicates that this organism is haploid; we can thus assign a chromosome number of at least 11 to this yeast. $C$. stellatoidea (lane 3 ) has eight bands. Once again, band 3 appears to be a doublet, so that we arrive at a chromosome number of nine. The pattern of $C$. stellatoidea should be compared with those of $C$. albicans strains 1840 in lane 4 and SC5314 in lane 5, which have very different karyotypes from each other and from $C$. stellatoidea. Different $C$. albicans isolates vary greatly in their 

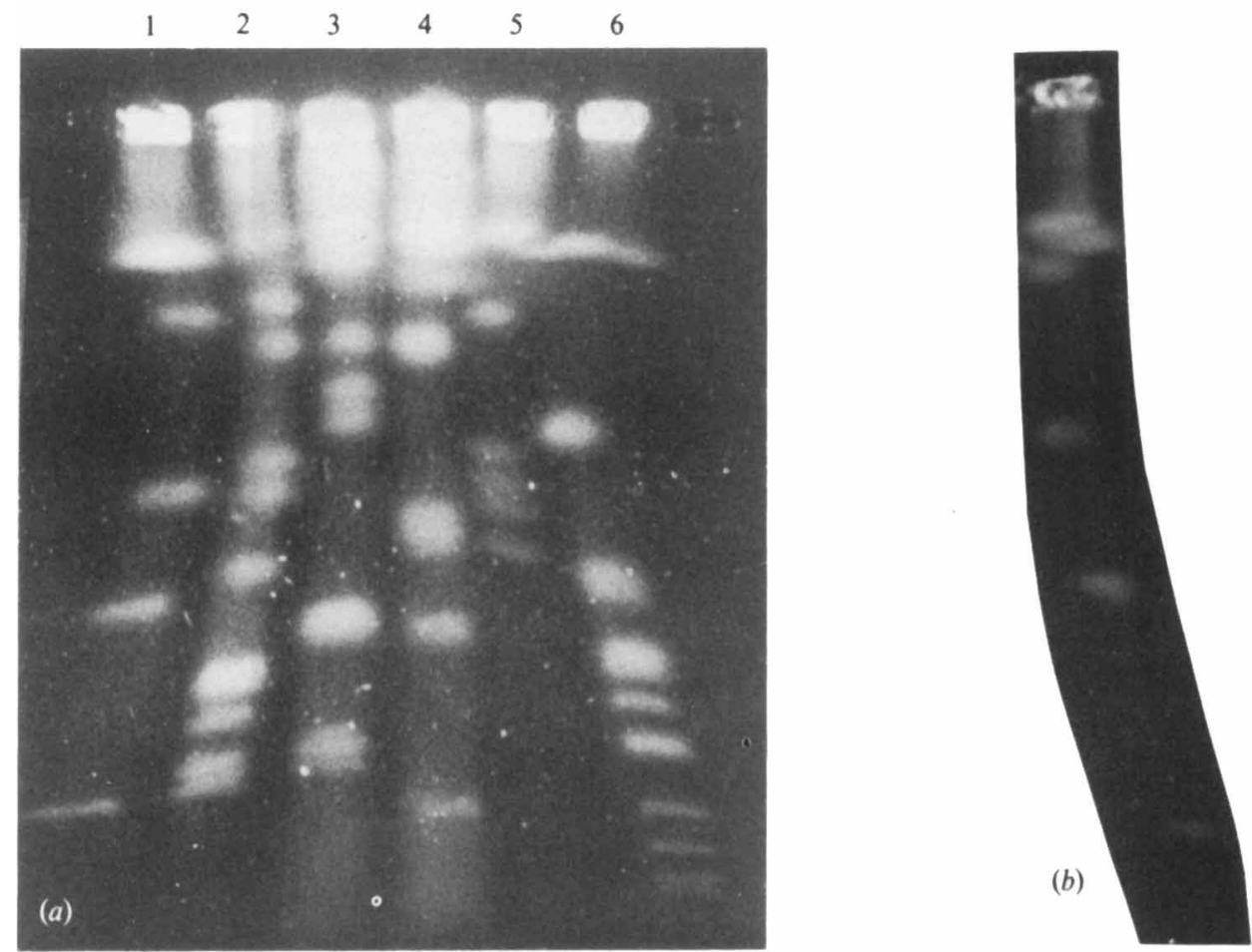

Fig. 2. (a) Electrophoretic karyotypes of several Candida strains. A $1 \cdot 2 \%$ agarose gel was run at a $70 \mathrm{~s}$ pulse rate at $300 \mathrm{~V}$ for $21 \mathrm{~h}$. Lane 1, C. guilliermondii NUM20; lane 2, C. glabrata 81/011; lane 3, C. stellatoidea CP5Am5; lane 4, C. albicans 1840; lane 5, C. albicans SC5314; lane 6, Sacch. cerevisiae BB4.

(b) C. guilliermondii NUM20 in a different run (conditions as above).

electrophoretic karyotypes (see below), but $C$. stellatoidea, which is thought to be closely related to, if not a subspecies of, C. albicans, appears quite different from the general pattern. Our estimate of the number of chromosomes in the two species, however, is the same (see below). Lane 6 shows the pattern of Sacch. cerevisiae.

\section{C. albicans strains show polymorphism in chromosome band size}

Since $C$. albicans has no sexual cycle, the grouping of organisms into this species has been traditionally based upon morphological and nutritional characteristics. We have recently found, using restriction fragment length polymorphisms, that $C$. albicans does indeed appear to be a genetically homogeneous class for the most part (T. D'Souza, B. B. Magee \& P. T. Magee, unpublished). We therefore examined the karyotypes of a number of $C$. albicans isolates. Fig. $3(a)$ shows the electrophoretic karyotypes of four C. albicans isolates, including one from Japan. The figure shows that the $C$. albicans karyotype is quite variable, at least so far as the size of the chromosomes is concerned. Lanes 1 and 2 show FC18, a strain which has been used extensively in this and other laboratories for genetic analysis. FC18 has two bright bands in the lower part of the gel where SC5314 has four (lanes 3 and 4) less bright ones; we infer that these two bands in $\mathrm{FC} 18$ are doublets and the $\mathrm{FC} 18$ has four chromosomes in this size range. Band 3 of FC18 (equivalent to band 5 of SC5314) migrates more slowly, and both strains appear to have two bands in the relatively poorly resolved upper region of the gel. Lanes 5 and 6 show the electrophoretic karyotype of NUM63; this organism contains one very bright, broad band in the fast-moving region. This band is inferred to contain the four bands seen in SC5314. There is one band in the region of band 5, migrating more rapidly than in either FC18 or SC5314. A bove this band, the slowly moving region is resolved into four bands. Lanes 7 and 8 show SG483; this 

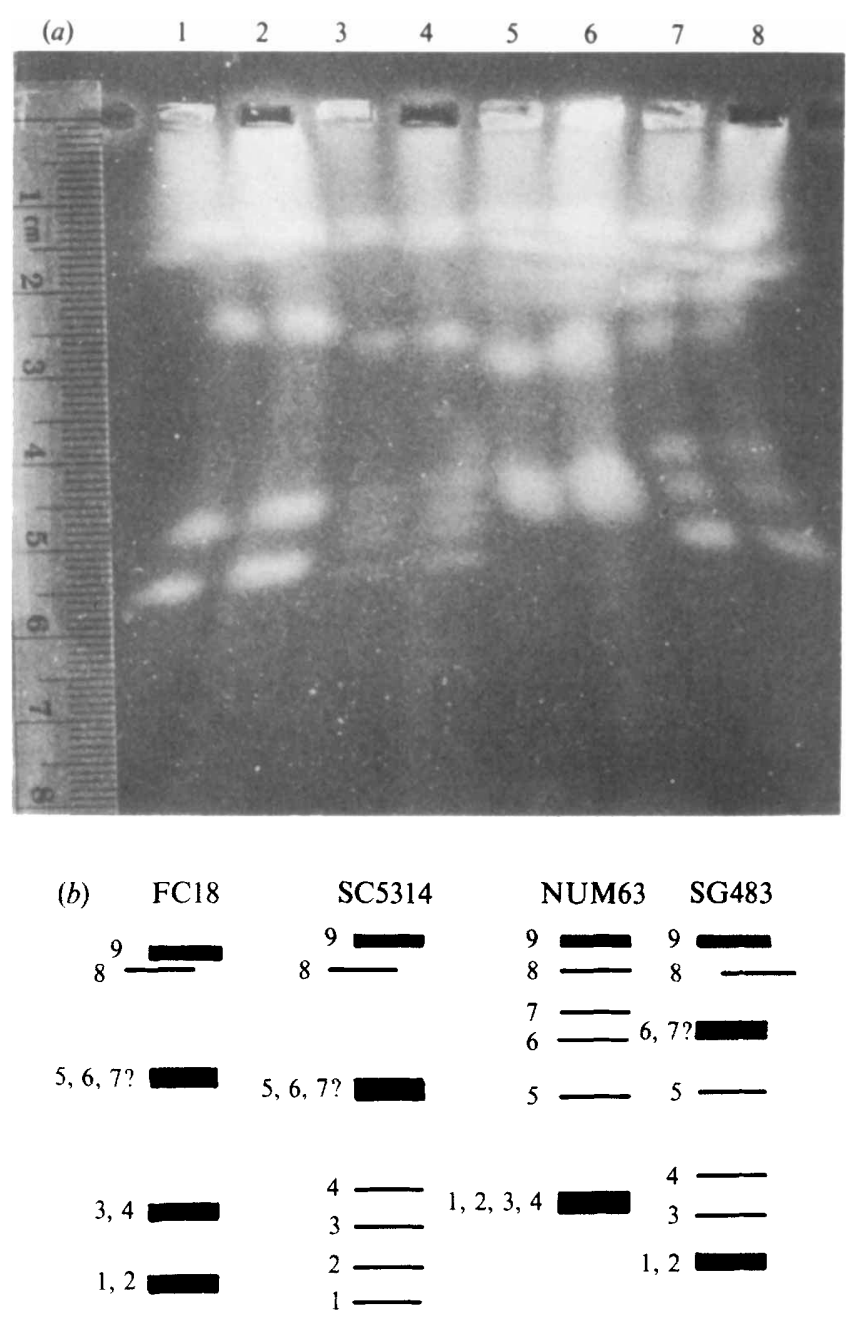

Fig. 3. (a) DNA from four strains of $C$. albicans was loaded on a $1 \%$ agarose gel, which was run at a $70 \mathrm{~s}$ pulse rate at $300 \mathrm{~V}$ for $21 \mathrm{~h}$. Lanes 1 and $2 \mathrm{FCl} 18$; lanes 3 and $4 \mathrm{SC} 5314$; lanes 5 and $6 \mathrm{NUM} 63$; lanes 7 and 8 SG483. (b) A schematic representation of the gel in $(a)$.

strain gives three bands below band 5 and three above it. We can thus, using all these strains, count nine chromosomal bands from $C$. albicans. Fig. 3(b) shows a schematic representation of the gel, with the bands numbered. The interpretation of which chromosomal bands are unresolved in some strains is, of course, tentative and will be discussed further below.

\section{DISCUSSION}

The results presented in this paper indicate that OFAGE is a useful technique for determining the electrophoretic karyotypes of a number of medically important yeasts. Of the six Candida species we have studied, five have, in general, very large chromosomes. $C$. guilliermondii has one of its six chromosomes in the size range of most Sacch. cerevisiae bands, while $C$. glabrata has a karyotype with a distribution not too different from Sacch. cerevisiae. In keeping with the taxonomic distinction among these species, the electrophoretic karyotypes are quite distinct. 
We have not conducted a survey of multiple representatives of most of these organisms, so we cannot comment on possible polymorphisms in the karyotypes, but if the variation from strain to strain in most Candida species is not greater that it is in C. albicans, OFAGE may be a rapid and easy way of identifying clinical isolates.

The genetic studies which have located at least five linkage groups in C. albicans make it of considerable interest to determine the chromosome number in this organism and to try to identify physically the chromosomes to which the linkage groups correspond. Our results show that the degree of polymorphism in the electrophoretic karyotypes of $C$. albicans is such that it is important to survey several strains to determine the chromosome number, since single bands in the karyotype of one strain will sometimes resolve in another. This phenomenon has been seen in Sacch. cerevisiae by Carle \& Olson (1985). It is also important, particularly in these yeasts with very large chromosomes, to experiment with conditions to resolve the material in the uppermost part of the gel. We have achieved enough resolution so that by comparing karyotypes of various strains, we can count nine bands.

Whether this is the definitive chromosome number for $C$. albicans is not clear at this time. There are several points which must be considered in evaluating these results. The first is that the band at the top of the gel is still bright in every case and may conceal two or more unresolved chromosomes. This, of course, would lead to an underestimate of the actual chromosome number. A second consideration is that the organism as usually isolated is diploid; thus, two bands which are resolved in one strain and unresolved in another may be chromosome homologues. Fortunately, C. albicans NUM63 is a haploid (or near haploid) isolate (T. Suzuki, B. B. Magee \& P. T. Magee, unpublished) and thus one can be reasonably confident that all bands resolved in this organism are non-homologues. The fact that the lower four chromosomes do not resolve in NUM63 allows for the possibility that they represent two pairs of homologues in SC5314, although the extreme brightness of the band in NUM63 does not support that idea. It seems most likely, therefore, that $C$. albicans has at least nine chromosomes. The issue of homologues versus non-homologues can be resolved by blotting with cloned DNA to determine whether any two of the bands contain the same genetic information. It is important to note that these same considerations hold for the electrophoretic karyotypes of any of the normally diploid yeasts.

Fig. 3(b) shows our interpretation of the relationships between the bands in Fig. 3(a). We have tried to make the most plausible assignments, based upon brightness, of all the bands resolved in the various species. Each strain in Fig. $3(a)$ is an authentic C. albicans by the API assimilation test and by rDNA restriction enzyme analysis (T. D'Souza, B. B. Magee \& P. T. Magee, unpublished). We have made the assumption that all C. albicans will contain the same number of chromosomes. This assumption can be tested by Southern blots, and such experiments are in progress.

These results constitute a significant step toward improving the genetic system in C. albicans. We now have available parasexual genetics (Kakar et al., 1983; Poulter et al., 1981; Sarachek et al., 1981) including induced chromosome loss techniques (Hilton et al., 1985), whole cell genetic exchange (Suzuki et al., 1986) and transformation (Kurtz et al., 1986) as genetic tools. The information derived from these systems can now be correlated with electrophoretic bands corresponding to chromosomes; this should greatly facilitate the coordination of information gained from the various systems.

We thank Dr Donald Williamson of the National Institute for Medical Research, Mill Hill, London, UK, for introducing B. B. Magee to the OFAGE technique, Dr Maynard Olson for helpful advice, Drs Erik Rikkerink and Taka Suzuki for thoughtful discussions, and Drs Frank Odds and Alvin Rogers for the gift of Candida strains. This work was supported by NIH Grant AI16567.

\section{REFERENCES}

Carle, G. F. \& Olson, M. V. (1984). Separation of chromosomal DNA molecules from yeast by orthogonal-field-alternation gel electrophoresis. Nucleic Acids Research 12, 5647-5664.
CARle, G. F. \& Olson, M. V. (1985). An electrophoretic karyotype for yeast. Proceedings of the National Academy of Sciences of the United States of America 82, 3756-3760. 
Crandall, M. (1982). UV-induced mitotic cosegregation of genetic markers in Candida albicans: evidence for linkage. Current Genetics 7, 167-173.

Hilton, C., Markie, D., Corner, B., Rikkerink, E. \& Poulter, R. (1985). Heat shock induces chromosome loss in the yeast Candida albicans. Molecular and General Genetics 200, 162-168.

Kakar, S. N., Partridge, R. M. \& Magee, P. T. (1983). A genetic analysis of Candida albicans: isolation of a wide variety of auxotrophs and demonstration of linkage and complementation. Genetics 104, 241-255.

Kurtz, M. B., Cortelyou, M. W. \& Kirsch, D. R. (1986). Integrative transformation of Candida albicans using a cloned Candida $A D E 2$ gene. Molecular and Cellular Biology 6, 142-149.

ODDs, F. C. (1979) Candida and Candidosis. Leicester: Leicester University Press.

Poulter, R. \& Hanrahan, V. (1983). Conservation of genetic linkage in nonisogenic isolates of Candida albicans. Journal of Bacteriology 156, 498-506.
Poulter, R., Jeffery, K., Hibbard, M. J., Shepherd, M. G. \& Sullivan, F. A. (1981). Parasexual genetic analysis of Candida albicans by spheroplast fusion. Journal of Bacteriology 146, 833-840.

SaRacheK, A., RhoAds, D. D. \& Schwarzhof, R. H. (1981). Hybridization of Candida albicans through fusion of protoplasts. Archives of Microbiology 129, $1-8$.

SChWARTZ, D. C. \& CANTOR, C. R. (1982). Separation of yeast chromosome-sized DNAs by pulsed field gradient gel electrophoresis. Cell 37, 67-75.

SuzUkI, T., Rogers, A. J. \& MAGEe, P. T. (1986). Inter- and intra-species crosses between Candida albicans and Candida guilliermondii. Yeast 2, 53-58. Whelan, W. L., Partridge, R. M. \& Magee, P. T. (1980). Heterozygosity and segregation in Candida albicans. Molecular and General Genetics 180, 107113. 